\section{Breeding for PVY resistance in tobacco LJ911 using CRISPR/Cas9 technology}

\author{
Ren Ruyi ${ }^{1}$, Zheng Qiang ${ }^{1}$, Ni Futai ${ }^{2}$, Jin Qiu ${ }^{1}$, Wan Xiuqing ${ }^{3}$ and \\ Wei Jicheng ${ }^{1 *}$
}

\begin{abstract}
Potato virus Y (PVY) is one of the main diseases of tobacco (Nicotiana tabacum). Recent studies indicated that the Va gene (Ntab0942120) determines the susceptibility of the crop to PVY, and that the Va gene product interacts with the PVY genome-linked protein (VPg) to initiate the PVY genome translation process, which ultimately leads to the systemic infection of tobacco by the virus. In this research, tobacco cultivar LJ911 was used as receptor material for gene editing. Gene Va was knocked out through CRISPR/Cas9 technology, and transgene-free homozygous edited plants in the T1 generation were established. Pathology tests indicated that the edited plants had gained PVY resistance. Therefore, the edited materials generated in this study represent potentially useful genetic resources for breeding of PVY- resistant tobacco.
\end{abstract}

Keywords: PVY; tobacco; CRISPR/Cas9; Va; resistance breeding

\section{INTRODUCTION}

Potato virus Y (PVY) has been listed among the top 10 economically and scientifically important plant viruses. It causes one of the most damaging diseases of cultivated tobacco around the world. Due to the lack of resistant germplasm in cultivated tobacco and the rather recent understanding of the susceptibility mechanism of tobacco to PVY, the progress of research on tobacco breeding for PVY resistance was delayed. The resistance of tobacco Virgin A Mutant (VAM) against PVY was obtained by UV-induced mutagenesis (Koelle 1961). This trait is inherited as a single recessive gene $(v a)$, which has been introgressed into several tobacco genotypes such asTennessee86 and NC744 (Chaplin et al. 1980, Athow et al. 1987). The comprehension of the genetic mechanism related to PVY resistance in plants began with the discovery that the genome-linked protein (VPg) of PVY could interact with elF4E (iso) in the host plant (Schaad et al. 2000), followed by the identification of the recessive resistance gene eIF4E in many plants (Yeam et al. 2007, Wang and Krishnaswamy 2012). It has been shown that VPg can interact with elF4E, mimicking the $5^{\prime}$-cap structure of messenger RNAs. Later, an elF4E member (S10760, or Ntab0942120) was identified as the " $V a$ " gene by genome-wide transcriptome analysis and genetic mapping in tobacco (Julio et al. 2015).

The CRISPR/Cas9 (clustered regularly interspaced short palindromic repeats/ CRISPR-associated Cas9) system (Mali et al. 2013) is being used as the third generation of gene editing technology. In comparison with the previous two generations of gene editing technology, namely ZFNs (Zinc finger nucleases) and
Crop Breeding and Applied Biotechnology 21(1): e31682116, 2021 Brazilian Society of Plant Breeding. Printed in Brazil http://dx.doi.org/10.1590/198470332021v21n1a6 
TALENs (transcription activator-like effector nucleases), it has the advantages of simplicity and efficiency. The CRISPR/ Cas 9 system has been widely used in the breeding of tobacco traits, including quality, disease resistance, yield, etc (Sun et al. 2017, Jia et al. 2017, Cai et al. 2018, Li et al. 2018, Sánchez-León et al. 2018, Liu et al. 2019, Zhu et al. 2019, Wu et al. 2020). LJ911 is the main flue-curled tobacco variety with poor PVY resistance in Heilongjiang province of China. In this research, the CRISPR/Cas9 system is used to knock out the susceptible gene Va to improve PVY resistance of the generated materials, which can serve as a potential resistance source in tobacco breeding.

\section{MATERIAL AND METHODS}

\section{Material}

Seeds of tobacco variety LJ911 and strain PVYN were provided by the Mudanjiang Tobacco Research Institute of Heilongjiang province, China. All primers were synthesized by the Shanghai Shenggong Biology Engineering Technology Service (the sequences were outlined in Table 1). The base vector PTX41T for CRISPR/Cas9-mediated editing of gene Va was provided by Dr. Deng Lei from the Institute of Genetics and Developmental Biology of the Chinese Academy of Sciences.

\section{Expression vector construction for Va gene editing and genetic transformation of tobacco}

Two 19 base pair target sequences were determined by sequence analysis of the first exon of Va (Appendix I). According to the construction procedure proposed by Deng Lei (Deng et al. 2018), plasmid pTX41T was used as template; the two gRNA target sequences were incorporated into the PCR forward and reverse primers, Va-g1F/Va-g2R. After Bsal digestion, the PCR product was introduced into the expression vector PTX41 (Figure 1). The resulting expression vector was named pTX41-VaE and subsequently introduced into the Agrobacterium tumefaciens strain EHA105 by electroporation. The axenic tobacco LJ911 seedlings were used as transformation explant material. Tobacco transformation was carried out using the Agrobacterium-mediated method with leaf-disc explants. T0 events were generated using kanamycin selection in MS medium.

\section{Detection of edited plants}

DNA was extracted from TO transgenic lines by the CTAB method and PCR was carried out with the vector-specific primers VaE-T-F/VaE-T-R to identify the transformants. Using DNA isolated from the transformants as template, PCR was carried out with Va gene-specific primers VaE-F/VaE-R to amplify a region of the first exon. The edited plants were identified after sequencing the PCR products; then the specific mutation status of the edited plants was determined after TA cloning and sequencing.

Table 1. Primer sequence. Primers for introducing Cas9 single guide sequence (target sequence is underlined)

\begin{tabular}{|c|c|}
\hline Va-g1F & ATATATGGTCTCGTTTGGTGGATGAATCTGATGATAGTTTTAGAGCTAGAAATAGC \\
\hline Va-g2R & ATTATTGGTCTCGAAACAGAATTCTCTAATGGATGCCCAAACTACACTGTTAGATTC \\
\hline \multicolumn{2}{|c|}{ Primers for detection of vector T-DNA insert construction detection: } \\
\hline NtVa-g1F & GTGGATGAATCTGATGATAG \\
\hline NtVa-g2R & AGAATTCTCTAATGGATGCC \\
\hline \multicolumn{2}{|c|}{ Specific primer for Va fragment amplification: } \\
\hline VaE-F & CACGAAAATGGCAGAGGAAG \\
\hline Nt4350-F & GATGAAGTAGAGAAACCGGC \\
\hline Nt4350-R & GAAACCAAAGTAAGGGGTAGC \\
\hline Nt2370-F & CGAAGACTAATACTCGTGAG \\
\hline Nt2370-R & CAAAGTAAGGGGTAGCGTACG \\
\hline $\mathrm{Nt5} 460-\mathrm{F}$ & GCTATAACAAGCTAATAATGAC \\
\hline
\end{tabular}




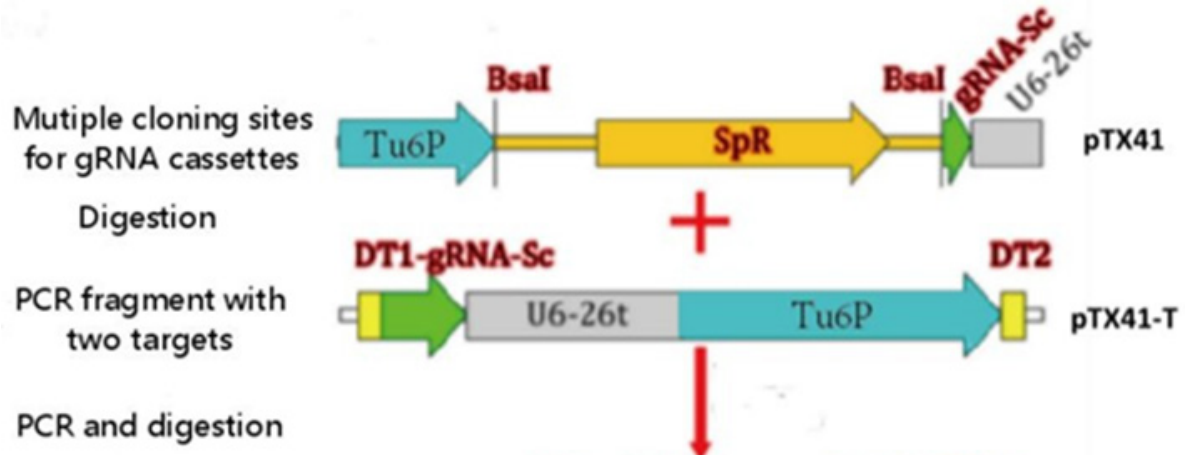

Two gRNA cassettes

(Final construct)

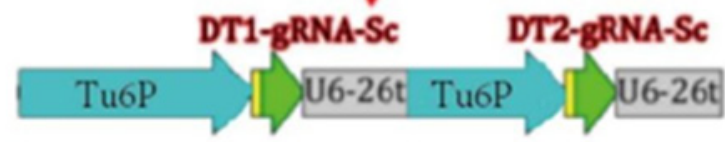

Figure 1. Construction procedure of editing vector for expression of two sgRNA sequences. Manual search for 23-bp target sites (5'-N ${ }^{\prime}$ NGG-3') within exons of genomic DNA sequences of genes of interest. Design primers: Replace 19-nt N in the forward primers with the 19-nt target sequences upstream of PAM (NGG), and 19-nt $\mathrm{N}$ in the reverse primers with reverse complement sequences of the 19-nt target sequences upstream of PAM(NGG).

g1F: ATATATGGTCTCGTTTGNNNNNNNNNNNNNNNNNNNGTTTTAGAGCTAGAAATAGC.

g2R: ATTATTGGTCTCGAAACNNNNNNNNNNNNNNNNNNNCCAAACTACACTGTTAGATTC. Carry out PCR reactions (with pTX41T as template). Set up digestion (Bsa I) and ligase reactions (PTX41 and PCR fragment). Transform E. coli competent cells and identify correct clones by colony PCR and verify them by sequencing.

To obtain Cas9 transgene-free T1 progeny plants, DNA was isolated from the T1 tobacco plants and PCR was carried out with the vector-specific primers VaE-T-F/VaE-T-R. The PCR- negative plants were identified as Cas9-free edited lines.

\section{Detection of off-target effect}

Va belongs to the elF4E gene family, which contains 11 members in tobacco. To evaluate the off-target effect, the potential target sequences were identified by homologous alignment (Clustal Omega) of target sequences among the 11 members and specific primers were designed to amplify the potential target members. PCR amplification and sequencing were conducted to evaluate the possibility of off-target editing in the edited plants.

\section{Pathology test of Cas9 transgene-free edited plants}

Fresh PVY-infected tobacco leaves were frozen in liquid nitrogen and ground thoroughly, then diluted with water at a ratio of 1:100. PVY was inoculated on the Cas9-free edited plants by friction, and susceptibility symptoms were assessed after 20 days.

\section{RESULTS AND DISCUSSION}

Indels occurred mainly $3 \mathrm{bp}$ upstream of the PAM sequence in the edited plants

The genetic transformation of tobacco was carried out with leaf-disk as explant mediated by Agrobacterium tumefaciens, and 56 resistant seedlings were finally obtained after kanamycin resistance screening, by which 43 transformants were identified. To identify edited seedlings, 13 transformants were selected randomly after DNA isolation and PCR amplification with a pair of specific primers, VaE-F/VaE-R, flanking the target sequences. Seven edited seedlings (named LJ911E1 - LJ911E7) were obtained after sequencing and comparison with the wild type sequence. The detailed mutation information is given in Table 2.

Although the editing vector expresses gRNAs targeting two Va gene target sequences, the indels occurred only in the first target sequence. It is not clear why the 2nd target sequence was not edited. Except for LJ911E6, the indels occurred mainly $3 \mathrm{bp}$ upstream of the PAM sequence, which is the predicted Cas9 cleavage site. In Table 2, LJ911E1 
Table 2. Mutations created in tobacco LJ911

\begin{tabular}{|c|c|c|}
\hline Line ID Target sites & & Indels \\
\hline $\begin{array}{l}\text { WT } \\
\text { LJ911E1 }\end{array}$ & $\begin{array}{l}\text { GTGGATGAATCTGATGATACGG } \\
\text { GTGGATGAATCTGATGAATACGG }\end{array}$ & +1 \\
\hline LJ911E2 & GTGGATGAATCTG---ATACGG & -3 \\
\hline LJ911E4 & GTGGATGAATCTGAT-ATACGG & -1 \\
\hline LJ911E5-1 & GTGGATGAAT ATACGG & -6 \\
\hline LJ911E6-2 & GTGGATGAATCTGAT-ATACGG & -1 \\
\hline LJ911E7-1 & GTGGATGAA-ATACGG & -7 \\
\hline LJ911E7-2 & GTGGATGAATCTG---ATACGG & -3 \\
\hline
\end{tabular}

(CGG: PAM sites).

is the only homozygous edited plant; the wild type sequence (not listed in the Table) was also detected in LJ911E2, LJ911E3, and LJ911E4, indicating that all of them were heterozygous. There was a 3bp deletion in LJ911E2, which led to the deletion of only one amino acid. According to the sequence analysis, the position was not in the functional domain of elF4E responsible for binding the cap structure of messenger RNA (Kinkelin et al. 2012); so, its homozygous progenies might not affect the pathogenicity of the protein. Biallelic mutations occurred on the two homologous chromosomes in LJ911E5, LJ911E6 and LJ911E7. Therefore, LJ911E1 is the best material to evaluate the effect of Va gene editing on virus resistance in subsequent studies, by eliminating the T-DNA component by segregation in its progeny plants.

\section{Va editing confers PVY resistance to cultivated tobacco LJ911}

To obtain Cas9-free edited tobacco, 15 progenies of LJ911E1 were used for PCR amplification with the vector-specific primers (VaE-T-F/VaE-T-R) after DNA isolation. No vector was detected in the seven seedlings, indicating that they were Cas9 transgene-free. The Cas 9 transgene-free homozygous edited tobacco was selected and inoculated with PVY virus by friction inoculation and the vaccination results were assessed at 20 DPI. In Figure 2, the control (wild-type) tobacco leaves had turned yellow and the vein color had changed, indicating that the disease had entered into the stage of vein necrosis; this indicated immunity of the Va edited tobacco lines to PVY.

The presence of the Cas9 transgene may lead to continuous cleavage of the genomic sequences, posing a potential risk to the trait stability. The present study demonstrated the establishment of pure homozygous Cas9 transgene-free offsprings and their PVY resistance was confirmed by pathology tests. However, the resistant material can currently not be used in agricultural production in China, owing to the regulation of gene editing products. The spontaneous mutation and gene-edited crops are genetically indistinguishable. In the United States, gene-edited products are not regulated as genetically modified organisms. Several edited crops using CRISPR/Cas9 technology are already applied in commercial production (Waltz 2016, Dong et al. 2020); but this kind of product is still restricted by the regulatory framework for GMO of the EU. So far, there is no specific regulation clause in China, so at present the edited material can only be treated as a reserve of resistance germplasm resources.

\section{Very low ratio of off-target effect in edited plants}

For off-target analysis, four potential target members (Ntab0384350, Ntab0982370, Ntab0285450 and Ntab0285460) were identified by analyzing the homologous sequences of the elF4E family in tobacco. Primers corresponding to the four potential target members were designed according to the target sequence.

The PCR products were sequenced and the results of off-target analysis were summarized (Table 3 ). Only edited plant LJ911E7 had an off-target cleavage effect in the homologous region of Ntab0384350. Consequently, LJ911E7 should not be used as alternative material for subsequent breeding, due to this off-target effect. This effect has been a potential problem since the invention of gene-editing technology. There are many factors causing the off-target effect, of which the most important is the sequence homology between the potential off-target and the desired on-target effects. The low off-target effect probability in this study may be due to the mismatches between the corresponding regions of non-target members and sgRNA. 

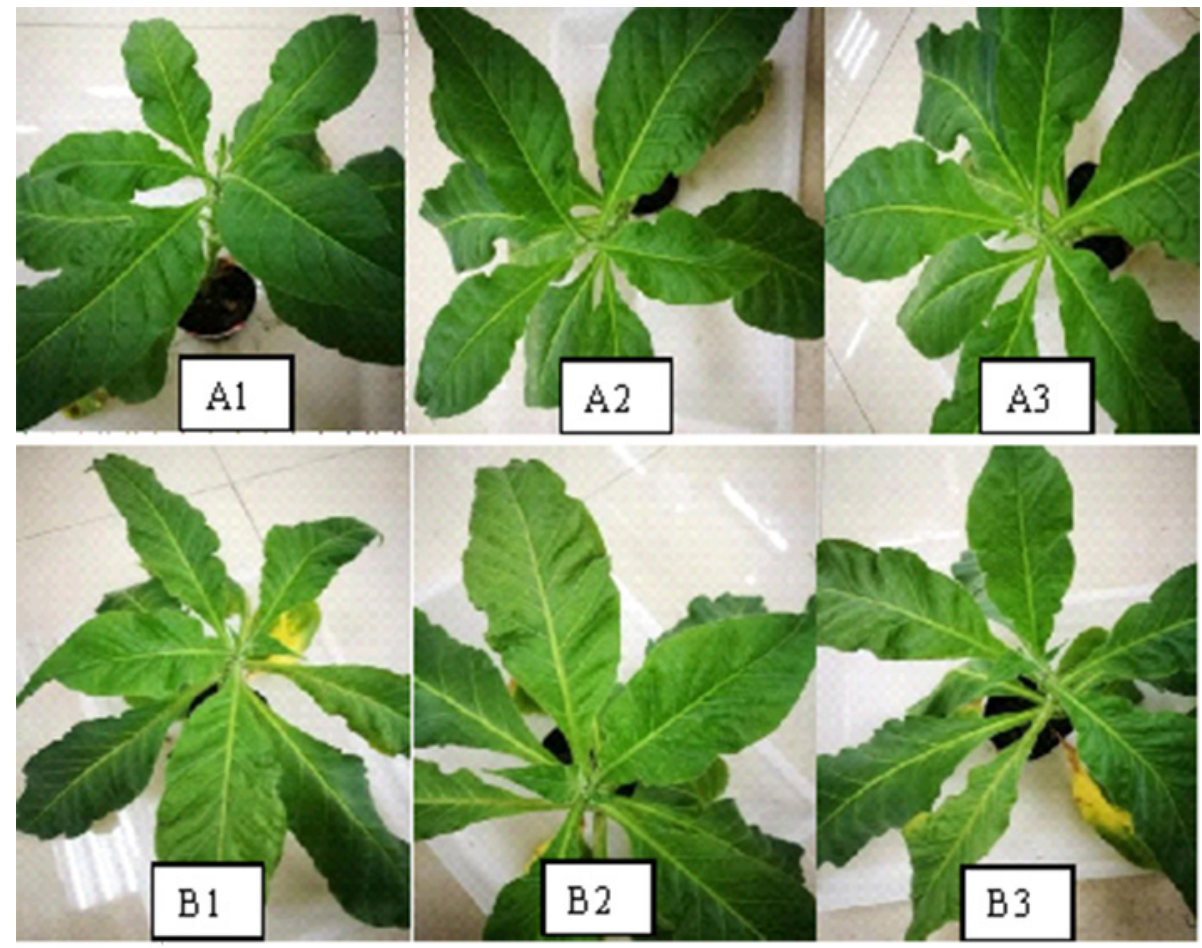

Figure 2. PVY inoculation. A1-A3: The vaccination results of Va edited tobacco; B1-B3: The vaccination results of control tobacco.

Table 3. Off-target effects

\begin{tabular}{|c|c|c|c|c|}
\hline Edited seedlings & Ntab0982370 & Ntab0384350 & Ntab0285450 & Ntab285460 \\
\hline LJ911E1 & $x$ & $x$ & $x$ & $x$ \\
\hline LJ911E2 & $x$ & $x$ & $x$ & $x$ \\
\hline LJ911E3 & $x$ & $x$ & $x$ & $x$ \\
\hline LJ911E4 & $x$ & $x$ & $x$ & $x$ \\
\hline LJ911E5 & $x$ & $x$ & $x$ & $x$ \\
\hline LJ911E6 & $x$ & $x$ & $x$ & $x$ \\
\hline
\end{tabular}

( $\mathbf{v}$ : off-target effect; $\mathbf{x}$ : no off-target effect detected).

\section{CONCLUSION}

The knockout of Va gene confers PVY resistance to cultivated tobacco, indicating that Va can be used as a suitable target for improving PVY resistance in tobacco.

\section{ACKNOWLEDGMENTS}

The auhors wish to thank Dr. Deng Lei for providing the vector system for gene editing, and are indebted to the Tobacco Monopoly Administration of Heilongjiang Province, China [grant number HN201503], for funding this work.

\section{REFERENCES}

Athow KL, Laviolette FA, Wilcox JR and Abney TS (1987) Registration of

"TN86" burley tobacco. Crop Science 27: 365-366

Cai Y, Chen L, Liu X, Guo C, Sun S, Wu C, Jiang B, Han T and Hou W

(2018) CRISPR/Cas9-mediated targeted mutagenesis of GmFT2a delays flowering time in soya bean. Plant Biotechnology Journal 16: 176-185.

Chaplin JF, Burk LG, Gooding GV and Powell NT (1980) Registration of NC 744 tobacco germplasm. Crop Science 20: 677.

Deng L, Wang H, Sun C, Li Q, Jiang H, Du M, Li CB and Li C (2018) Efficient 


\section{R Ruyi et al.}

generation of pink-fruited tomatoes using CRISPR/Cas9 system. Journal of Genetics and Genomics 45: 51-54.

Dong OX, Yu S, Jain R, Zhang N, Duong PQ, Butler C, Li Y, Lipzen A, Martin JA, Barry KW, Schmutz J, Tian L and Ronald PC (2020) Marker-free carotenoid-enriched rice generated through targeted gene insertion using CRISPR-Cas9. Nature Communications 11: 1178

Jia H, Zhang Y, Orbović V, Xu J, White FF, Jones JB and Wang N (2017) Genome editing of the disease susceptibility gene CsLOB1 in citrus confers resistance to citrus canker. Plant Biotechnology Journal 7: 817-823.

Julio E, Cotucheau J, Decorps C, Volpatti R, Sentenac C, Candresse T and Dorlhac de Borne F (2015) A eukaryotic translation initiation factor $4 \mathrm{E}$ (elF4E) is responsible for the "va" tobacco recessive resistance to potyviruses. Plant Molecular Biology Reporter 3: 609-623.

Kinkelin K, Veith K, Grünwald M and Bono F (2012) Crystal structure of a minimal elF4E-Cup complex reveals a general mechanism of elF4E regulation in translational repression. RNA 9: 1624-1634

Koelle G (1961) Genetics analyse einer Y-Virus-(Rippenbräune) resistenten mutante der tabaksorte virgin A. Züchter 31: 71-71

Li S, Liu S, Liu Y, Lu H, Tan Y, Huang J, Wei P and Shu Q (2018) Hrm-facilitated rapid identification and genotyping of mutations induced by CRISPR/ Cas9 mutagenesis in rice. Crop Breeding and Applied Biotechnology 18: 184-191.

Liu E, Zeng S, Zhu S, Liu Y, Wu G, Zhao K, Liu X, Liu Q, Dong Z, Dang X, Xie H, Li D, Hu X and Hong D (2019) Favorable alleles of GRAIN-FILLING RATE1 increase the grain-filling rate and yield of rice. Plant Physiology 181: 1207-1222.

Mali P, Aach J, Stranges PB, Esvelt KM, Moosburner M, Kosuri S, Yang L and Church GM (2013) CAS9 transcriptional activators for target specificity screening and paired nickases for cooperative genome engineering. Nature Biotechnology 9: 833-838.

Sánchez-León S, Gil-Humanes J, Ozuna CV, Giménez MJ, Sousa C, Voytas DF and Barro F (2018) Low-gluten, nontransgenic wheat engineered with CRISPR/Cas9. Plant Biotechnology Journal 16: 902-910

Schaad MC, Anderberg RJ and Carrington JC (2000) Strain-specific interaction of the tobacco etch virus Nla protein with the translation initiation factor elF4E in the yeast two-hybrid system. Virology 2: 300-306.

Sun Y, Jiao G, Liu Z, Xin Z, Li J, Guo X, Du W, Du J, Francis F, Zhao Y and Xia L (2017) Generation of high-amylose rice through CRISPR/ Cas9-mediated targeted mutagenesis of starch branching enzymes. Frontiers in Plant Science 8: 298.

Waltz E (2016) Gene-edited CRISPR mushroom escapes US regulation. Nature 7599: 293.

Wang A and Krishnaswamy S (2012) Eukaryotic translation initiation factor 4E-mediated recessive resistance to plant viruses and its utility in crop improvement. Molecular Plant Pathology 13: 795-803.

Wu N, Lu Q, Wang P, Zhang Q, Zhang J, Qu J and Wang N (2020) Construction and analysis of GmFAD2-1A and GmFAD2-2A soybean fatty acid desaturase mutants based on CRISPR/Cas9 Technology. International Journal of Molecular Sciences 21: 1104

Yeam I, Cavatorta JR, Ripoll DR, Kang BC and Jahn MM (2007) Functional dissection of naturally occurring amino acid substitutions in elF4E that confers recessive potyvirus resistance in plants. Plant Cell 9: 2913-2928.

Zhu Y, Lin Y, Chen S, Liu H, Chen Z, Fan M, Hu T, Mei F, Chen J, Chen L and Wang F (2019) CRISPR/Cas9-mediated functional recovery of the recessive rc allele to develop red rice. Plant Biotechnology Journal 17: 2096-2105.

\section{APPENDIX I - SEQUENCE OF VA FRAGMENT}

$>\mathrm{Va}$

CACGAAAATGGCAGAGGAAGCTGAGAAATTGCGGGTAGATGAAGTAGAAGTAGTCG ACGATGGACCTGAAGAAGGAGAAATTGTGGATGAATCTGATGATACGGCGTCGTATT TGGGCAAAGAAATCAAACCTAAGCATCCATTAGAGAATTCTTGGACTTTTTGGTTTG ATAATCCTATGGCTAAATCTAGACAAGCTGCTTGGGGCAGTTCCCTTCGCGAACTTTA CACTTTTTCCACTGTCGAAGATTTTTGGGGGT 